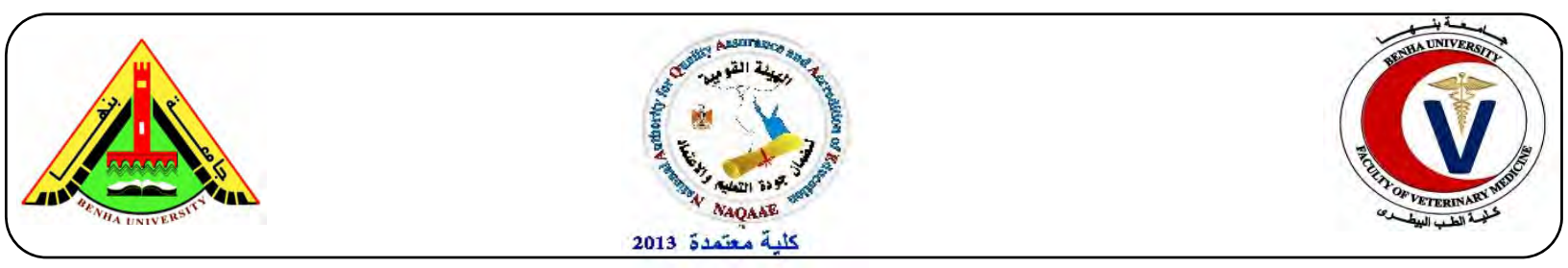

\title{
A trial to improve stability and immunogenicity of inactivated NDV vaccine with paraffin oil adjuvant using aluminum stearate.
}

\author{
El-Bagoury, G.F. '; Nasr, M.H.M.'; El-Habbaa, A.S. 1; Hala, M. El-Makaky² \\ ${ }^{1}$ Department of Virology, Fac. of Vet. Med., Benha Univ., Egypt. \\ ${ }^{2}$ Animal Health Research Institute (Benha branch), Egypt. \\ ${ }^{2}$ Veterinary Serum and Vaccine Research Institute, Abbasia, Cairo, Egypt
}

\section{A B S T R A C T}

Inactivated Newcastle disease virus (NDV) vaccines were prepared using paraffin oil, paraffin oil and aluminum stearate (A1.St.) and Montanide oil ISA 71VG as adjuvants. All experiments gave the priority to the inactivated NDV vaccines with paraffin oil adjuvant and Al.St. $4-5 \mathrm{mg} /$ dose including stability (drop test, centrifugation and real time test) and viscosity (dynamic and relative) over NDV vaccine adjuvant with paraffin oil alone that showed reduced stability, however NDV vaccine with paraffin oil and Al.St. $6 \mathrm{mg} / \mathrm{dose}$ and $7 \mathrm{mg} /$ dose showed high values of viscosity indicating hard injectability. Chicks vaccinated with inactivated NDV vaccines with paraffin oil adjuvant and Al.St. (4, 5, 6 and $7 \mathrm{mg} /$ dose) gave higher cell mediated immune response (lymphocyte blastogenesis and macrophage activity), humoral immune responses (using haemagglutination inhibition test) and $100 \%$ protection upon challenge experiment in comparison to that vaccinated with NDV vaccine with paraffin oil adjuvant alone that showed $93.3 \%$ and $86.66 \%$ protection for vaccines used immediately after preparation and the others used after one year of storage at $4^{\circ} \mathrm{C}$, respectively. Chicks vaccinated with NDV vaccines with paraffin oil adjuvant and Al.St showed slightly lower values of both cellular and humoral immune responses than that of NDV vaccine adjuvant with Montanide TM ISA 71VG. In conclusion, inactivated NDV vaccine prepared with paraffin oil adjuvant and aluminum stearate $(4 \mathrm{mg} / \mathrm{dose}$ and $5 \mathrm{mg} / \mathrm{dose})$ were superior for that adjuvant on paraffin oil alone, as they improved stability and potency of the vaccine, and were with the same quality to that of NDV vaccine adjuvant on the imported Montanide ISA $71 \mathrm{VG}$.

Keywords: Inactivated NDV vaccine, HI test, Paraffin oil, Aluminum stearate.

(http:// www.bvmj.bu.edu.eg)

(BVMJ-28(1): 199-209, 2015)

\section{INTRODUCTION}

$\mathrm{N}$ ewcastle disease (ND) is the most devastating disease affecting poultry especially in large scale production causing severe economic losses due to high mortality which may reach $100 \%$ in very virulent strains of ND Virus (Alexander, 2003). ND Virus (NDV) belongs to family Paramyxoviridae, genus Avulavirus (Miller et al., 2010). Vaccination is the most effective method to control and prevent ND in poultry, thus, innovative vaccines, vaccination regimen and routes are necessary for the control of the disease (OIE, 2009). In Egypt, control policy of ND depended for many years mainly upon vaccination with live vaccines; however, the inactivated aluminum hydroxide gel adjuvanted NDV vaccine was produced (Nadia et al., 1992, Daoud et al., 2002). Serological evidence indicated that oil emulsion inactivated NDV vaccine induced higher haemagglutinating inhibiting antibody titer as well as more persistent immunity than aluminum hydroxide vaccine (Box and Furminger, 1975 and Stone el al., 1980). High quality oil emulsion vaccine should be stable, with low viscosity to ease injectability and produce suitable haemagglutinating inhibiting antibody titer in vaccinated birds. The quality of the adjuvant emulsion has direct impact on the safety and efficacy of 
the vaccine, so good physical properties of the inactivated oil vaccine could in turn increase the immune response to this vaccine (stone et al., 1983). Aluminum stearate, an emulsifying agent and stabilizer added to the oil adjuvant of the prepared vaccine to produce higher antibody titers with longer duration of immunity and additionally will be non-toxic to the host, was long used to improve oil emulsion vaccines for inactivated Avian Encephalomyelitis vaccine (Wang el al., 1995), inactivated combined NDV and Egg Drop Syndrome virus vaccine (Zheng el al., $1995)$ inactivated Infectious Bursal disease virus vaccine (Long et al, (2009) and inactivated avian influenza disease virus (H5N1 subtype) produced by Harbin veterinary research institute (HVRI, 2006) which were stable for many months, safe and effective. So the final goal of this study was to prepare an inactivated NDV vaccine with improved quality using paraffin oil adjuvant and aluminum stearate, and its evaluation in comparison to other prepared vaccines using paraffin oil adjuvant alone or the imported ISA 71VG.

\section{MATERIAL AND METHODS}

\subsection{New castle disease virus:}

\subsubsection{La Sota strain:}

La Sota strain of NDV obtained from Veterinary Serum and Vaccine Research Institute (VSVRI), which was supplied from the central veterinary Lab., Weighbridge, England. The virus is lentogenic and has a titer of $1011 \mathrm{Egg}$ Infective Dose 50\% (EID50)/ml and was for preparation of inactivated NDV vaccines under study.

\subsubsection{Virulent strain of NDV:}

Velogenic viscerotropic NDV (VVNDV) field isolate (Reda and Sheble, 1976), was obtained from the department of poultry viral vaccine, VSVRI, Abbasia, Cairo. Its titer was $10^{6} \mathrm{EID}_{50} /$ Dose. It was used in challenge experiment of vaccinated chicks.

\subsection{Embryonated Chicken Eggs (ECE)} and Experimental chicks:

Fertile SPF-ECE and chicks were purchased from the SPF egg project, Kom
Oshim, EL-Fayoum Governorate. The eggs were used for propagation and titration of ND viruses and ensuring of completion of virus inactivation. The chicks were floor reared, fed on balanced commercial poultry ration, and kept under strict hygienic measures throughout the experiment. They were used in studying potency of the prepared vaccines.

\subsection{Serum samples:}

Serum samples were collected from experimental chicks weekly till $10^{\text {th }}$ week post vaccination (WPV) then every 2 weeks till the $28^{\text {th }} \mathrm{WPV}$. The sera were inactivated at $56^{\circ} \mathrm{C}$ for 30 minutes, and then stored at $20^{\circ} \mathrm{C}$ until used in $\mathrm{HI}$ test.

\subsection{Whole blood samples:}

Jugular blood samples from experimental chicks were collected with anticoagulant (Heparin 20-40 IU/ml) for evaluation of cell mediated immune response using lymphocyte blastogenesis and macrophage activity tests.

\subsection{Vaccine preparation:}

\subsubsection{Propagation and titration of La Sota NDV in SPF-ECE:}

The seed virus for vaccine preparation was propagated via allantoic sac in 9-11 days old SPF-ECE $(0.2 \mathrm{ml} / \mathrm{egg})$ according to Allan et al. (1973), which was rapidly detected in embryonic fluid with $10 \%$ washed chicken red blood cell suspension in saline using Rapid Plate Haemagglutination test according to Anon, (1971), then titrated according to Reed and Meunch (1938). The Aqueous phase used for vaccine formulation is adjusted to have a titer $10^{11}$ EID $_{50} /$ dose of the seed virus.

\subsubsection{Inactivation of the propagated NDV}

\section{La Sota strain:}

It was done according to Hofstad et al., (1963) using formalin (37\%) Analar, BDH that was added to the harvested allantoic fluid in a final concentration of $0.1 \%$, left for 18 hours at room. Safety of the inactivated virus was tested by injecting 0.2 $\mathrm{ml}$ of the inactivated fluid into 9-11 days ECE through allantoic sac for 3 blind passages. Inoculated ECE were observed 
for 6 days for embryo deaths and harvested fluids were tested by the rapid HA test.

\subsubsection{Preparation of inactivated NDV oil adjuvant vaccines:}

\subsubsection{Inactivated NDV vaccine with paraffin oil adjuvant (NDV-paraffin oil):}

It was prepared as water in oil $(\mathrm{W} / \mathrm{O})$ emulsion according to Daoud et al., (2002) with both the oil soluble surfactant Span 80 (MP Biomedical Inc., France, Lot. No. 1553J) and the aqueous soluble surfactant Tween 80 (Sigma-Aldrich, Germany, Lot No. 085k0096) were added to the oil phase (Paraffin oil, white X300 oil quality FDAIAL USP Lot No. 05200 Mobil) and the aqueous phase (inactivated virus suspension) respectively. Then the aqueous-phase was added to the oil-phase at a ratio 1:3 drop by drop with continuous mixing for 30 minutes after last drop to give homogenized emulsion and the hydrophilic lipophilic balance (HLB) was adjusted to 7.0 as previously described by Schick, (1966) and Stone et al., (1978).

\subsubsection{Inactivated NDV vaccine with paraffin oil adjuvant and aluminum stearate (NDV-aluminum Stearate):}

It was prepared according to Daoud et al., (2002) in which water in oil emulsion (W/O), with both the oil soluble surfactant (span 80) and the aqueous soluble surfactant (tween 80) were added to the oil phase (Paraffin oil) in addition to aluminum stearate (Al.St., Morgan specialty Chemicals Company, Egypt, Batch. No: 81926612,) in different concentrations separately $(4 \mathrm{mg} /$ dose, $5 \mathrm{mg} / \mathrm{dose}, 6 \mathrm{mg} /$ dose and $7 \mathrm{mg} / \mathrm{dose})$, then the aqueous-phase (Inactivated virus suspension) was added and the hydrophilic lipophilic balance (HLB) was adjusted to 7.0 as previously described by (Schick, 1966; Stone, 1988).

\subsubsection{Inactivated NDV vaccine with Montanide ISA71 adjuvant (NDV- ISA71):}

It was prepared as water in oil $(\mathrm{W} / \mathrm{O})$ emulsion using Montanide ISA71VG
(SEPPIC S.A, Paris, France, Bach No. T21931, Product code 36514P) at a ratio of $30 / 70 \quad(\mathrm{v} / \mathrm{v})$ aqueous /oil ratio. Manufacturing process was carried out according to the standard protocol of SEPPIC and manufacture instruction.

\subsection{Experimental design:}

A number of 620 of one-day-old specific pathogen free (SPF) chicks were divided into 60 birds used for evaluation of safety of the prepared vaccines (10 chick/vaccine) and the other 560 birds were divided into 14 group (40 chicks/ each), each chick in the vaccinated groups inoculated at 21 days of their age subcutaneously with $0.5 \mathrm{ml}$ of the prepared vaccine either after two weeks of preparation (experiment 1) or after one year of storage (experiment 2) for studying potency of the prepared vaccines. Challenge of experimental chicks was done at 21 days post vaccination for calculation of protection percent (as shown in table 1).

\subsection{Evaluation of the prepared inactivated} NDV oil emulsion vaccines:

\subsubsection{Evaluation of physical properties:}

Type of the oil emulsion (drop test), relative and dynamic viscosity and stability of the vaccine emulsions were examined according to Stone et al. (1978) and Brugh et al. (1983).

\subsubsection{Quality control:}

2.7.2.1. Sterility test:

It was applied to confirm that the prepared vaccines were free from bacterial, and fungal contamination by inoculation into nutrient agar, thioglycolate broth, and Saburaouds glucose agar that were incubated at $37^{\circ} \mathrm{C}, 37^{\circ} \mathrm{C}$ and $25^{\circ} \mathrm{C}$, respectively for 72 hours and mycoplasma on PPLO that was incubated at at $37^{\circ} \mathrm{C}$ for 14 days.

\subsubsection{Safety test in chicks:}

Safety of the prepared inactivated NDV oil emulsion vaccines were examined in groups of 3 weeks old chicks (10 chicks/vaccine), inoculated with $1 \mathrm{ml}$ (double dose) of each prepared vaccine subcutaneous at the nap of the neck. These chicks were observed for 2 weeks 
Table (1): Chick groups used in the designed experiments.

\begin{tabular}{|c|c|c|c|}
\hline \multicolumn{2}{|c|}{ Chick Group } & $\begin{array}{l}\text { Number } \\
\text { of birds }\end{array}$ & Experiment \\
\hline \multirow{7}{*}{ 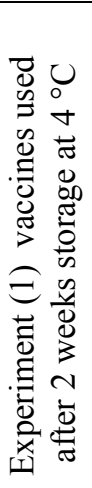 } & G1 & 40 & Chicks vaccinated with inactivated NDV vaccine with paraffin oil \\
\hline & & & adjuvant and Al.st. $4 \mathrm{mg}$ /dose, immediately after preparation. \\
\hline & $\mathrm{G} 2$ & 40 & $\begin{array}{l}\text { Chicks vaccinated with inactivated NDV vaccine with paraffin oil } \\
\text { adjuvant and Al.st. } 5 \mathrm{mg} / \text { dose, immediately after preparation. }\end{array}$ \\
\hline & G3 & 40 & Chicks vaccinated with inactivated NDV vaccine with paraffin oil \\
\hline & G4 & 40 & Chicks vaccinated with inactivated NDV vaccine with paraffin oil \\
\hline & G5 & 40 & $\begin{array}{l}\text { adjuvant and Al.st. } 7 \mathrm{mg} / \text { dose, immediately after preparation. } \\
\text { Chicks vaccinated with inactivated NDV vaccine with paraffin oil } \\
\text { adjuvant only, immediately after preparation. }\end{array}$ \\
\hline & G6 & 40 & $\begin{array}{l}\text { Chicks vaccinated with inactivated NDV vaccine with Montanide Тм } \\
\text { ISA } 71 \text { VG adjuvant, immediately after preparation. }\end{array}$ \\
\hline \multirow{8}{*}{ 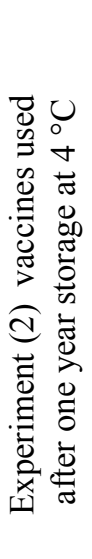 } & G7 & 40 & Chicks kept as non-vaccinated control. \\
\hline & G8 & 40 & Chicks vaccinated with inactivated NDV vaccine with paraffin oil \\
\hline & G9 & 40 & $\begin{array}{l}\text { adjuvant and Al.st. } 4 \mathrm{mg} \text { /dose, after one year of manufacture. } \\
\text { Chicks vaccinated with inactivated NDV vaccine with paraffin oil } \\
\text { adjuvant and Al.st. } 5 \mathrm{mg} / \text { dose, after one year of manufacture. }\end{array}$ \\
\hline & G10 & 40 & $\begin{array}{l}\text { Chicks vaccinated with inactivated NDV vaccine with paraffin oil } \\
\text { adjuvant and Al.st. } 6 \mathrm{mg} / \text { dose. }\end{array}$ \\
\hline & G11 & 40 & $\begin{array}{l}\text { Chicks vaccinated with inactivated NDV vaccine with paraffin oil } \\
\text { adjuvant and Al.st. } 7 \mathrm{mg} / \text { dose, after one year of manufacture. }\end{array}$ \\
\hline & G12 & 40 & $\begin{array}{l}\text { Chicks vaccinated with inactivated NDV vaccine with paraffin oil } \\
\text { adjuvant only, after one year of manufacture. }\end{array}$ \\
\hline & G13 & 40 & $\begin{array}{l}\text { Chicks vaccinated with inactivated NDV vaccine with Montanide }{ }^{\mathrm{TM}} \text { ISA } \\
71 \mathrm{VG} \text { adjuvant, after one year of manufacture. }\end{array}$ \\
\hline & G14 & 40 & Chicks kept as non-vaccinated control. \\
\hline 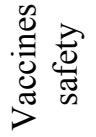 & G15 & 60 & $\begin{array}{l}\text { Chicks divided into subgroups } 10 \text { chicks/subgroup for testing safety of } \\
\text { each vaccine. }\end{array}$ \\
\hline
\end{tabular}

for signs of local reaction or appearance of clinical signs. After 5 days of inoculation, some birds were subjected to post mortem examinations to detect pathological lesions.

\subsubsection{Potency of the prepared vaccines:}

2.7.2.3.1. Evaluation of cell mediated immune response of chicks:

\subsection{Evaluation of lymphocyte} blastogenesis using XTT assay with tetrazolium dye:

Separation of lymphocytes from blood samples was applied according to Lucy, (1977) and Lee, (1984), viability of separated lymphocytes was determined according to Mayer et al., (1974) depending on the following formula:

Number of cell $/ \mathrm{ml}=$

cells counted in 32 squares $\times 10 \times 1000 x$ dilution 4
Viable lymphocytes were adjusted to a concentration of $5 \times 10^{6}$ cells $/ \mathrm{ml}$ suspended in RPMI-1640 (Roswer park Memorial Institute, Flow Lab. UK) tissue culture medium with L-glutamine containing $10 \%$ foetal calf serum and Evaluation of lymphocyte blastogenesis using XTT assay was performed according to Scudiero et al., (1988).

\subsection{Evaluation of Macrophage activity using candida albicans:}

Phagocytic percent and phagocytic index were carried out according to Richardson and Smith, (1981) and calculated as follow: Phagocytic percentage $=$

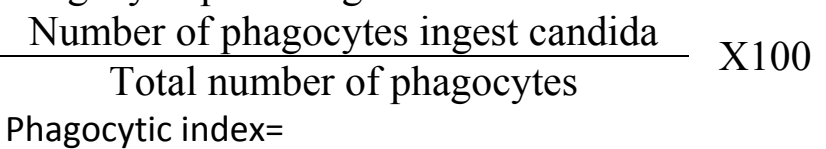

Total number of phagocytes which ingest more than two candida 
Total number of phagocytes which ingest candida All prepared inactivated NDV vaccines

\subsection{Studying humoral immune response using $\mathrm{HI}$ test:}

Haemagglutination inhibition (HI) test was carried out following the recommendation of OIE-Manual, (2004).

\subsection{Challenge test:}

Chick groups (vaccinated and un vaccinated control) were challenged 21 days post vaccination by $0.5 \mathrm{ml} /$ bird of virulent viscerotropic velogenic NDV containing $10^{6.5} \mathrm{EID}_{50} / \mathrm{ml}$, by $\mathrm{I} / \mathrm{M}$ route. The challenged birds were observed for 15 days post inoculation, serum samples were collected from them and dead birds through this time were recorded and examined for post-mortem lesions.

Protection \% =

Number of survived birds

Total number of challenged birds X 100

\section{RESULTS}

\subsection{Evaluation of physical properties:}

From table (2), the prepared NDV vaccines proved to be water-in-oil (w/o) emulsion type using drop test. Inactivated NDV vaccines adjuvant with paraffin oil and aluminum stearate at different concentrations and montanide ISA 71VG were stable using centrifugation test while that adjuvant with paraffin oil alone was unstable. Although stability of the prepared NDV vaccines using real time test differed at $4^{\circ} \mathrm{C}, \quad 25^{\circ} \mathrm{C}$ and $37^{\circ} \mathrm{C}$ storage temperatures, all vaccines were stable at $4^{\circ} \mathrm{C}$ storage temperature for 52 weeks post manufacture except for NDV vaccine adjuvant with paraffin oil alone which was less stable. All the prepared NDV vaccines showed acceptable relative viscosity, however NDV vaccines adjuvant with paraffin oil and Al.st $6 \mathrm{mg} /$ dose and $7 \mathrm{mg} /$ dose showed prolonged flow time.

3.2. Quality control:

3.2.1. Sterility test: were sterile and free from bacterial, mycoplasma and fungal contamination.

\subsubsection{Safety test in chicks:}

The prepared inactivated NDV oil emulsion vaccines were safe for inoculated chicks with neither local reaction nor clinical signs on examination.

\subsection{Potency of the prepared vaccines:}

Potency of the prepared inactivated NDV vaccines was studied for both NDV vaccines immediately after their preparation (Experiment 1) and after one year storage at $4{ }^{\circ} \mathrm{C}$ (Experiment 2).

\subsubsection{Cell mediated immune response of chicks vaccinated with NDV vaccines immediately after their manufacture (Experiment 1):}

3.3.1.1. Evaluation of lymphocyte blastogenesis using XTT assay with tetrazolium dye:

It was noticed that, significant increase in lymphocyte proliferation expressed by optical density were increased to reach maximum level in chicks $14^{\text {th }}$ day PV for all types of prepared NDV vaccines compared with that of control non vaccinated one. Inactivated NDV vaccines with paraffin oil adjuvant and aluminum stearate $4 \mathrm{mg} /$ dose and $7 \mathrm{mg} /$ dose and inactivated NDV vaccines with montanide TMISA 71 showed the highest lymphocyte proliferation compared with other inactivated NDV vaccines, as shown in table (3).

\subsubsection{Evaluation of Macrophage activity using candida albicans:}

It was observed that, both phagocytic percent and phagocytic index were significantly increased to reach maximum values during the $14^{\text {th }}$ day post vaccination in chicks vaccinated with all types of inactivated NDV vaccines compared with the control non vaccinated chicks. Inactivated NDV vaccines with paraffin oil adjuvant and aluminum stearate $4 \mathrm{mg} / \mathrm{dose}$, $5 \mathrm{mg} /$ dose and $7 \mathrm{mg} /$ dose and inactivated NDV vaccines with Montanide TMISA 71 
showed the highest phagocytic $\%$ and phagocytic index compared with other inactivated NDV vaccines, as shown in table (4).

Table (2): Physical properties examined for experimentally prepared oil emulsion NDV vaccines.

\begin{tabular}{|c|c|c|c|c|c|c|c|}
\hline \multirow{3}{*}{$\begin{array}{c}\text { Type of inactivated } \\
\text { NDV vaccine adjuvant }\end{array}$} & \multirow{3}{*}{$\begin{array}{l}\text { Emulsion type } \\
\text { (Drop test) }\end{array}$} & \multicolumn{4}{|c|}{ Emulsion stability } & \multicolumn{2}{|c|}{ Viscosity } \\
\hline & & \multirow[b]{2}{*}{ Centrifugation } & \multicolumn{3}{|c|}{ Real time test } & \multicolumn{2}{|c|}{ (Rheology test) } \\
\hline & & & $25^{\circ} \mathrm{C}$ & $37^{\circ} \mathrm{C}$ & $4^{\circ} \mathrm{C}$ & $\begin{array}{c}\text { Relative } \\
\text { (sec) }\end{array}$ & $\begin{array}{l}\text { Dynamic } \\
\text { (Mpa.s) }\end{array}$ \\
\hline Paraffin oil adjuvant with Al.st $4 \mathrm{mg} /$ dose & Water in oil & Stable & $\leq^{*} 2$ & $\leq 1$ & $>52$ & ${ }^{* * *} 17.4$ & ${ }^{* * *} 39.17$ \\
\hline Paraffin oil adjuvant with Al.st $5 \mathrm{mg} /$ dose & Water in oil & Stable & $\leq 2$ & $\leq 2$ & $>52$ & 24.73 & 43.65 \\
\hline Paraffin oil adjuvant with Al.st $6 \mathrm{mg} / \mathrm{dose}$ & Water in oil & Stable & $\leq 4$ & $\leq 4$ & $>52$ & 28.1 & 57.27 \\
\hline Paraffin oil adjuvant with Al.st $7 \mathrm{mg} / \mathrm{dose}$ & Water in oil & Stable & $\leq 26$ & $\leq 4$ & $>52$ & 35.63 & 68.06 \\
\hline Paraffin oil adjuvant only & Water in oil & Unstable & $\leq 2$ & $\leq 1$ & $\leq 26$ & 24.8 & 38.09 \\
\hline Montanide $^{\mathrm{TM}}$ ISA 71 & Water in oil & Stable & $\leq 4$ & $\leq 2$ & $>52$ & 9.26 & 26.56 \\
\hline
\end{tabular}

${ }^{*}$ Stability for weeks.

${ }^{* *}$ Flow time per second.

${ }^{* * *}$ European pharmacopeia acceptances limits of dynamic viscosity which ranged between $12 \geq R \leq 56$ mpa.s.

Table (3): Results of cell mediated immune response of chicks vaccinated with the prepared inactivated NDV vaccines determined by lymphocyte blastogenesis using XTT reagent.

\begin{tabular}{|c|c|c|c|c|c|c|}
\hline \multirow{2}{*}{$\begin{array}{l}\text { Chick } \\
\text { Groups }\end{array}$} & \multicolumn{6}{|c|}{$\begin{array}{c}\text { Cell proliferation expressed by optical density } \\
\text { days post vaccination }\end{array}$} \\
\hline & $3^{\text {th }}$ & $5^{\text {th }}$ & $7^{\text {th }}$ & $14^{\text {th }}$ & $21^{\text {th }}$ & $28^{\text {th }}$ \\
\hline G1 & ${ }^{*} 0.785$ & 1.3845 & 1.732 & 2.2325 & 1.982 & 0.3749 \\
\hline G2 & 0.890 & 1.003 & 1.89 & 1.978 & 1.434 & 0.3074 \\
\hline G3 & 0.875 & 1.4985 & 1.683 & 1.943 & 1.813 & 0.4274 \\
\hline G4 & 0.960 & 1.254 & 1.774 & 2.375 & 1.27 & 0.5689 \\
\hline G5 & 0.2597 & 0.768 & 0.805 & 1.512 & 1.1335 & 0.5984 \\
\hline G6 & 0.707 & 1.269 & 1.7155 & 2.521 & 2.23 & 0.927 \\
\hline G7 & 0.2657 & 0.3405 & 0.142 & 0.11075 & 0.1795 & 0.1049 \\
\hline
\end{tabular}

G1: Chicks vaccinated with inactivated NDV vaccine with paraffin oil adjuvant and Al.st. $4 \mathrm{mg} /$ dose.

G2: Chicks vaccinated with inactivated NDV vaccine with paraffin oil adjuvant and Al.st. $5 \mathrm{mg} /$ dose.

G3: Chicks vaccinated with inactivated NDV vaccine with paraffin oil adjuvant and Al.st. $6 \mathrm{mg} /$ dose.

G4: Chicks vaccinated with inactivated NDV vaccine with paraffin oil adjuvant and Al.st. $7 \mathrm{mg} /$ dose.

G5: Chicks vaccinated with inactivated NDV vaccine with paraffin oil adjuvant only.

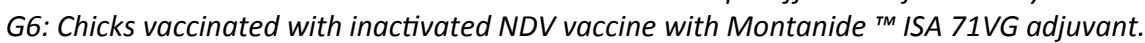

G7: Chicks kept as non-vaccinated control.

Table (4): Comparative evaluation of Macrophage activities in chicks vaccinated with inactivated NDV oil emulsion vaccines using candida albicans.

\begin{tabular}{ccccccccc}
\hline \multirow{2}{*}{$\begin{array}{c}\text { Chick } \\
\text { Groups }\end{array}$} & \multicolumn{6}{c}{ Macrophage activity evaluated at days post vaccination } \\
\cline { 2 - 8 } & $3^{\text {rd }}$ & $5^{\text {th }}$ & $7^{\text {th }}$ & $14^{\text {th }}$ & $3^{\text {rd }}$ & $5^{\text {th }}$ & $7^{\text {th }}$ & $14^{\text {th }}$ \\
\cline { 2 - 8 } G1 & $52.3 \%$ & $60.7 \%$ & $65 \%$ & $88.8 \%$ & 0.37 & 0.44 & 0.68 & 0.75 \\
G2 & $44.4 \%$ & $55.3 \%$ & $66.66 \%$ & $87.5 \%$ & 0.28 & 0.56 & 0.71 & 0.88 \\
G3 & $33.3 \%$ & $50.9 \%$ & $55.5 \%$ & $62.5 \%$ & 0.40 & 0.60 & 0.77 & 0.83 \\
G4 & $22.2 \%$ & $44.4 \%$ & $73.3 \%$ & $88.8 \%$ & 0.30 & 0.60 & 0.66 & 0.90 \\
G5 & 16.6 & $29.41 \%$ & $44.4 \%$ & $58.3 \%$ & 0.22 & 0.36 & 0.40 & 0.70 \\
G6 & $55.5 \%$ & $66.6 \%$ & $75 \%$ & $88.8 \%$ & 0.50 & 0.66 & 0.75 & 0.86 \\
G7 & $6.25 \%$ & $6.66 \%$ & $7.4 \%$ & $5.84 \%$ & 0.1 & 0.07 & 0.09 & 0.16 \\
\hline
\end{tabular}

G1: Chicks vaccinated with inactivated NDV vaccine with paraffin oil adjuvant and Al.st. $4 \mathrm{mg} /$ dose.

G2: Chicks vaccinated with inactivated NDV vaccine with paraffin oil adjuvant and Al.st. $5 \mathrm{mg} /$ dose.

G3: Chicks vaccinated with inactivated NDV vaccine with paraffin oil adjuvant and Al.st. $6 \mathrm{mg} /$ dose.

G4: Chicks vaccinated with inactivated NDV vaccine with paraffin oil adjuvant and Al.st. $7 \mathrm{mg} /$ dose.

G5: Chicks vaccinated with inactivated NDV vaccine with paraffin oil adjuvant only.

G6: Chicks vaccinated with inactivated NDV vaccine with Montanide ${ }^{\mathrm{TM}}$ ISA 71 VG adjuvant.

G7: Chicks kept as non-vaccinated control. 


\subsection{Studying humoral immune response:}

Humoral immune response was studied in chicks vaccinated with NDV vaccines immediately after their manufacture (Experiment 1) and after one year storage at $4^{\circ} \mathrm{C}$ (Experiment 2). It was observed that chicks vaccinated with NDV vaccine with paraffin oil adjuvant and aluminum stearate (experiments 1 and 2) gave higher mean $\log _{2} \mathrm{HI}$ antibody titer than that of chicks vaccinated with NDV vaccine with paraffin oil adjuvant alone especially in the $3^{\text {rd }}$ WPV. Chicks vaccinated with inactivated NDV vaccines with paraffin oil adjuvant and aluminum stearate gave slightly insignificant lower mean $\log _{2} \mathrm{HI}$ antibody titer than that of chicks vaccinated with NDV vaccine adjuvant with Montanide TM ISA $71 \mathrm{VG}$ especially in the $3^{\text {rd }}$ WPV. Duration of immune response was prolonged to $28^{\text {th }} \mathrm{WPV}$ for all prepared NDV vaccines in experiment 1 except for
NDV vaccine with paraffin oil adjuvant only which prolonged in a suitable mean $\log _{2} \mathrm{HI}$ antibody titer for 16 weeks only. All these results were shown in tables $(5,6)$.

\subsection{Protection Rate:}

All vaccinated chicks showed 100\% protection percent upon challenge 21 days post vaccination for both NDV vaccines used immediately after their manufacture (Experiment 1) and that used after one year storage at $4{ }^{\circ} \mathrm{C}$ (Experiment 2), except for chicks vaccinated with NDV vaccine adjuvant with paraffin oil only that showed $93.3 \%$ protection for chicks vaccinated with NDV vaccines immediately after their manufacture (Experiment 1) and $86.66 \%$ for chicks vaccinated with NDV vaccines used after one year storage at $4^{\circ} \mathrm{C}$ (Experiment 2). These results were compared with zero protection percent of the control non vaccinated and challenged group as shown in tables $(7,8)$.

Table (5): Mean $\log _{2}$ antibody titer in sera from chicks vaccinated with prepared inactivated oil emulsion NDV vaccine using HI test.

\begin{tabular}{|c|c|c|c|c|c|c|c|c|c|c|c|c|c|c|c|c|c|}
\hline Chick & \multicolumn{17}{|c|}{ Mean $\log _{2} \mathrm{HI}$ antibody titer weeks post vaccination } \\
\hline Groups & 1 & 2 & 3 & 4 & 5 & 6 & 7 & 8 & 9 & 10 & 12 & 14 & 16 & 18 & 20 & 24 & 28 \\
\hline G1 & 4 & 5.33 & 7.8 & 8.5 & 8.5 & 8 & 6.25 & 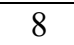 & 8.75 & 6.66 & & 75 & 7 & 4.5 & 3.25 & 4 & 3.33 \\
\hline $\mathrm{G} 2$ & 3.33 & 5.66 & 7.66 & 8.66 & 8.75 & 7.75 & 8.25 & 9.5 & 7 & 7.5 & 6.5 & 6.25 & 5.75 & 3.75 & 4.25 & 3.66 & 4.33 \\
\hline G3 & 3.66 & 6 & 7.5 & 8.25 & 8.5 & 8.25 & 7.66 & 9 & 7.75 & 6.66 & J & 7 & 6.5 & 4 & 3.5 & 4.25 & 3.33 \\
\hline G4 & 3. & 5.66 & 8 & 8. & 8.7 & 9. & 8 & 10 & 9.25 & 8.5 & 7.75 & 8.25 & 5.5 & 4.25 & 5 & 5.5 & 4.33 \\
\hline G5 & 3.33 & 5.33 & 7 & 7.33 & 7.25 & 6.66 & 7.66 & 7.25 & 6 & 6. & 5. & 1 & 3 & 2.25 & 2 & 2.66 & 2 \\
\hline G6 & 4.33 & 6.66 & 8.66 & 9.25 & 10.5 & 9.66 & 10.25 & 9.25 & 9.75 & 8.5 & 7.5 & 8 & 7.75 & 7 & 6.5 & 7 & 6.25 \\
\hline G7 & 0 & 0 & 0 & 0 & 0 & 0 & 0 & 0 & 0 & 0 & 0 & 0 & 0 & 0 & 0 & 0 & 0 \\
\hline
\end{tabular}

Table (6): Mean $\log _{2} \mathrm{HI}$ antibody titer in sera from chicks vaccinated with inactivated oil emulsion NDV vaccine after one year storage at $4^{\circ} \mathrm{C}$.

\begin{tabular}{|c|c|c|c|c|c|c|}
\hline \multirow{2}{*}{$\begin{array}{l}\text { Chick } \\
\text { Groups }\end{array}$} & \multicolumn{6}{|c|}{ Mean $\log _{2}$ HI antibody titer weeks post vaccination } \\
\hline & 1 & 2 & 3 & 4 & 5 & 6 \\
\hline G8 & 3.33 & 5 & 7.33 & 8 & 8.33 & 8.66 \\
\hline G9 & 2.7 & 5 & 7.66 & 7.66 & 8 & 8.33 \\
\hline $\mathrm{G} 10$ & 3 & 4 & 7.33 & 8.33 & 9 & 8.66 \\
\hline G11 & 3 & 5.66 & 7.66 & 8.66 & 9.33 & 9 \\
\hline G12 & 2.33 & 3.66 & 6.66 & 7 & 8 & 8.33 \\
\hline G13 & 4 & 5.7 & 9 & 9.66 & 10 & 10 \\
\hline G14 & 0 & 0 & 0 & 0 & 0 & 0 \\
\hline \multicolumn{7}{|c|}{$\begin{array}{l}\text { G8: Chicks vaccinated with inactivated NDV vaccine with paraffin oil adjuvant and Al.st. } 4 \mathrm{mg} / \text { dose. } \\
\text { G9: Chicks vaccinated with inactivated NDV vaccine with paraffin oil adjuvant and Al.st. } 5 \mathrm{mg} / \text { dose. } \\
\text { G10: Chicks vaccinated with inactivated NDV vaccine with paraffin oil adjuvant and Al.st. } 6 \mathrm{mg} / \text { dose. } \\
\text { G11: Chicks vaccinated with inactivated NDV vaccine with paraffin oil adjuvant and Al.st. } 7 \mathrm{mg} / \text { dose. } \\
\text { G12: Chicks vaccinated with inactivated NDV vaccine with paraffin oil adjuvant only. } \\
\text { G13: Chicks vaccinated with inactivated NDV vaccine with Montanide TM ISA 71VG adjuvant. } \\
\text { G14: Chicks kept as non-vaccinated control. }\end{array}$} \\
\hline
\end{tabular}


Table (7) Protection percentage in vaccinated and control non- vaccinated chicks in experiment (1) after challenge with VVNDV at 21 days post vaccination.

\begin{tabular}{ccccc}
\hline \multirow{2}{*}{$\begin{array}{c}\text { Chick } \\
\text { groups }\end{array}$} & \multicolumn{2}{c}{ Number of chicks } & \multirow{2}{*}{ Protection Percent } \\
\cline { 2 - 4 } G1 & 15 & Dead & Live & \\
G2 & 15 & 0 & 15 & $100 \%$ \\
G3 & 15 & 0 & 15 & $100 \%$ \\
G4 & 15 & 0 & 15 & $100 \%$ \\
G5 & 15 & 0 & 15 & $100 \%$ \\
G6 & 15 & 1 & 14 & $93.3 \%$ \\
G7 & 15 & 0 & 15 & $100 \%$ \\
\hline
\end{tabular}

Table (8) Protection percentage in vaccinated and control non-vaccinated chicks in experiment (2) after challenge with VVNDV at 21 days post vaccination .

\begin{tabular}{ccccc}
\hline Chick & \multicolumn{3}{c}{ Number of chicks } & \multirow{2}{*}{ Protection Percent } \\
\cline { 2 - 4 } groups & Challenged & Dead & Live & \\
\hline G8 & 15 & 0 & 15 & $100 \%$ \\
G9 & 15 & 0 & 15 & $100 \%$ \\
G10 & 15 & 0 & 15 & $100 \%$ \\
G11 & 15 & 0 & 15 & $100 \%$ \\
G12 & 15 & 2 & 13 & $86.66 \%$ \\
G13 & 15 & 0 & 15 & $100 \%$ \\
G14 & 15 & 15 & 0 & $0 \%$ \\
\hline
\end{tabular}

\section{DISCUSSION}

The standard requirements for water-in-oil $(\mathrm{W} / \mathrm{O})$ emulsion NDV vaccine in relation to its physical characters as emulsion viscosity, stability as well as potency evaluation had been studied. Examination of the physical properties of the prepared inactivated ND vaccine adjuvant with paraffin oil and aluminum stearate in different concentrations in comparison to NDV vaccine adjuvant with paraffin oil only and NDV vaccine adjuvant with Montanide ${ }^{\mathrm{TM}}$ ISA $71 \mathrm{VG}$. The quality of the adjuvant emulsion has direct impact on the efficacy and the safety of the vaccine. Physiochemical parameters of an emulsion include droplet test, conductivity, viscosity, particle size and stability at various temperatures (Lissant, 1984 and Salager, 2000).

Drop test revealed that all of the prepared vaccines were water-in-oil (w/o) emulsion type. Examination of the vaccine stability using centrifugation test, showed that all prepared NDV vaccines were stable except for inactivated NDV vaccine with Paraffin oil adjuvant only was unstable which was confirmed using real time test for evaluation of the period of keeping quality of vaccine as shelf life. The best physical properties of prepared vaccines from the stability point that using of aluminum stearate in concentrations of $4 \mathrm{mg} /$ dose, 5 $\mathrm{mg} /$ dose, $6 \mathrm{mg} /$ dose and $7 \mathrm{mg} /$ dose give long duration of stability equal or more than 52 weeks at $+4 \mathrm{C}^{\circ}$ with no separation as water release or oil release. The same results were obtained with inactivated NDV vaccine prepared with the imported Montanide $^{\mathrm{TM}}$ ISA $71 \mathrm{VG}$ adjuvant in comparison with the inactivated NDV vaccine adjuvant with paraffin oil adjuvant only without aluminum stearate (method of locally prepared NDV oil emulsion vaccine) which gave short duration of stability equal or less than 26 weeks, as presented in table (2). Concerning dynamic viscosity measured using capillary viscometer, European pharmacopeia, (2007) showed that the accepted limits of dynamic viscosity of water in oil emulsion vaccine using mineral oil ranged $[12 \leq \mathrm{R} \leq 56$ $\mathrm{mPa} . \mathrm{s}]$, the limits allow the vaccine could be easily injection. Results showed that using of concentration of $4 \mathrm{mg} /$ dose and 5 
$\mathrm{mg}$ /dose aluminum stearate were located within the accepted limits of dynamic viscosity, however $6 \mathrm{mg} /$ dose and $7 \mathrm{mg} /$ dose aluminum stearate were excluded from the limits of acceptance which lead to difficult in vaccine injection, higher fatigue of working and wasting of time specially in vaccination of large numbers of birds (Stone et al., 1983).

Relative viscosity expressed as flow time per second for inactivated ND vaccine adjuvant with paraffin oil and aluminum stearate $4 \mathrm{mg} /$ dose and $5 \mathrm{mg} /$ dose were 17.4 sec and $24.73 \mathrm{sec}$, respectively easily in injection, however inactivated NDV vaccine with paraffin oil and Al.st $6 \mathrm{mg} /$ dose and $7 \mathrm{mg} /$ dose showed prolonged flow time. This result agreed with Becher (1965) who observed that emulsion viscosity was found in inversely proportion with emulsion stability. Also the results of relative viscosity, where the fluid resistance of the prepared vaccines reflects on the injectability of vaccine, agreed with Berlin (1960) and Cessi and Nardelli, (1973).

Concerning the cellular immune response, results of lymphocytes blastogenesis as represented in table (3) revealed that a maximum response of $T$ cells expressed as optical density (OD) was recorded for different groups two weeks post vaccination, then the values become to decline till the end of the test, however birds of group (4) showed a higher stimulation (2.375) followed by group (1) as (2.2325) at $14^{\text {th }}$ day post vaccination. More confirmation of cellular immune response was achieved using Macrophage activity tests $(88.8 \%$ and $0.75-0.90$ as expressed by phagocytic percent and phagocytic index, respectively) were running parallel to the former test for the same groups at $14^{\text {th }}$ day post vaccination, results represented in table (4). Results of cellular immune response agreed with Timms and bracemell (1983) as they stated that once the humoral immune response becomes established, there is a corresponding decrease in the cellular immune response.
As the physical characters could in turn associate with increase the serological response (Stone et al., 1983), batches of the prepared NDV vaccines were subjected to study their efficacy in chicks vaccinated either immediately after preparation of the vaccines (experiment 1) and after one year storage (experiment 2).

Studying of the humoral immune response in chicks vaccinated with the prepared inactivated oil emulsion NDV vaccines (experiment 1) revealed that all the prepared vaccines regardless to the concentration of aluminum stearate produced higher mean titers of $\mathrm{HI}$ antibody from the first week post-vaccination (WPV) in all vaccinated chick groups in comparison to the slightly lower levels in chicks vaccinated with the prepared NDV vaccine with paraffin oil alone while the ND vaccine with the imported ISA $71 \mathrm{G}$ adjuvant gave the highest level of all, as shown in table (5). Comparatively, studying of the humoral immune response in chicks vaccinated with the prepared inactivated oil emulsion NDV vaccines (experiment 2) revealed that all the prepared vaccines gave high mean $\log _{2} \mathrm{HI}$ antibody titers from the first WPV and the maximum mean $\log _{2}$ titers as shown in table (6). These results were compared with that of prepared NDV vaccine with paraffin oil adjuvant only (local method) and prepared NDV vaccine adjuvant with the imported Montanide ISA $71 \mathrm{G}$. These results were correlated with the findings of Box and Furminger (1975) and Taha et al. (1991).

The protection against clinical disease was expected with the obtained HI antibody titers (tables 7 and 8) and application of challenge test on vaccinated chicks showed that protection percent for all prepared NDV vaccines were $100 \%$, except for groups vaccinated with NDV with paraffin oil-without any chemical substance showed that $93.3 \%$ in table (7) and $86.66 \%$ in table (8). Similar observations were obtained in this respect by Box and Furminger (1975) and Taha et al. (1991). 
In conclusion, it was found that the physical properties of prepared vaccine have a positive effect on the obtained serological responses as assured by challenge test and the prepared inactivated NDV vaccine with paraffin oil adjuvant and $4 \mathrm{mg} /$ dose and 5 $\mathrm{mg} /$ dose aluminum stearate were superior for that adjuvant on paraffin oil only, as they improved the physical characters and potency of the vaccine, and with the same quality to that of NDV vaccine adjuvant on the imported Montanide ISA adjuvant with high degree of stability as well as potency.

\section{REFERENCES}

Alexander, D.J. 2003. Newcastle Disease, other Avian Paramyxoviruses and Pneumovirus Infections. In: Diseases of Poultry, Saif, Y.M., Barnes, H.J., Glisson, J.R., Fadly, A.M., Mc Dougald, L.R., Swayne, D.E. (Eds.). $11^{\text {th }}$ Edn., Iowa State Press, Ames, pp: 63-99.

Allan, W.H., Lancaster, J.E., Toth, B. 1973. The Production and Use of Newcastle Disease Vaccines. FAO, Rome, Italy, Pages: 1115.

Anon, Y. 1971. Methods for Examining Poultry Biologics and for Identification and Quantifying Avian Pathogens. National Academy of Science, Washington, DC., USA., pp: 66.

Becher, P. 1965. Emulsion: Theory and Practice. 2nd ed. New York: Reinhold.

Berlin, B.S. 1960. Cross physical properties of emulsified influenza virus vaccine and adjuvants response. J.Immunol. 85: 81-89.

Box, P.G., Furminger, G.S. 1975:"Newcastle disease antibody levels in chickens after vaccination with oil emulsion adjuvanted killed vaccine".Vet.Res., 96: 108-111.

Brugh, M., Stone, H.D. Lupton, H.W. 1983. Comparison of inactivated Newcastle disease viral vaccines containing different emulsion adjuvants.-Am $\mathrm{J}$ Vet Res. 44(1): 72-5.

Cessi, D., Nardelli, L. 1973. Requirement for testing oil emulsion inactivated
Newcastle disease. Proc. 42nd symp. On requirement for poultry virus vaccines. Lyon.pp:326-328.

Daoud, A., Madkour, M.S., Abd El-Wains, N.A., Ensaf, K., Salwa, E. 2002. Studies for improvement of inactivated oil emulsion Newcastle disease virus vaccine, 6th Vet. Med. Zag. Conference (7-9 sept.2002), hurghada.

European pharmacopeia, 2007. $7^{\text {th }}$ rev 2010 p27.

Hofstad, M.S., Picken, J.C., Collins, Jr.K.E., Yoder, Jr.H.W. 1963. Immunogenicity of inactivated Newcastle disease virus preparation, Avian Disease, 7(4) p: 435-445.

HVRI, 2006. Harbin veterinary research institute of CAAS report of tests on the final product", 20, feb.2006. Harbin Weike Biotechnology Development Company, ID: Uu 06001- 0600206014"

Lee, L.F. 1984. Proliferative response of chicken $\mathrm{B}$ and $\mathrm{T}$ lymphocyte to mitogen. Vet. Med; 15: 44-52.

Lissant, K.J.L. 1984. Emulsions and emulsion technology, part III. In: Lissant Kenneth J, editor. Surfactant Science Series, 6:206-10.

Long, C., Mao, J.R., Xiao, S., Meng, C., Peng, C., Xiao, M.Y., Shung, Q. Z. 2009. BAFF enhance B-cell -mediated immune response and vaccineprotection against a very virulent IBDV in chicken. Vaccine, 27: 1393-1399.

Lucy, F.L. 1977. Chicken Lymphocyte stimulation by mitogenes .A microassay with whole blood cultures. Avian Dis.; 22,296-307.

Mayer, S.P., Ritts, G. D., Johnson, D. R. 1974. Phytohaemagglutinin induced leukocyte blastogenesis in normal and avian leucosis virus infection in chicken cells. Immunol; 27: 140-146.

Miller, P.J., Afonso, C.L., Spackman, E., Scott, M.A., Pedersen, J.C., Senne, D.A., Brown, J.D., Fuller, C.M., Uhart, M.M., Karesh, W.B., Brown, I.H., Alexander, D.J. and Swayne, D.E., 2010. Evidence for a new avian 
paramyxovirus serotype 10 detected in rockhopper penguins from the Falkland Islands. J. Virol., 84: 11496-11504.

Nadia, M.H., Ensaf, K., Fekria, E.B., Salwa, E.A., Elham, A.I., Salah, W., Nargis, M.B. 1992. "Trial for production of aluminum hydroxide gel and oily adjuvanted Newcastle disease vaccine in comparison with commercial imported oil vaccine " Assiut Vet. Med. J., 37: 53.

OIE "office international des epizooties" 2004. Manual of diagnostic tests and vaccines for terrestial animal Chapter 2.1.1.5 of Newcastle disease "OIE" Paris.

OIE "office international des epizooties" 2009. Newcastle Disease. In: Manual of Diagnostic Tests and Vaccines for OIE Terrestrial Animals 2009, OIE (Ed.). Chapter 2.3.14, 1, OIE, Paris, France, pp: 576-589.

Reda, I.M., Sheble, A. 1976. Isolation and characterization of a local velogenic viscerotropic strain of Newcastle disease virus. M.M. Emara, Cairo University, Egypt.

Reed, L.J. and Muench, H. 1938. A simple method of estimating 50 percent end points. Am. J. Hyg., 27: 493-495.

Richardson, M.D., Smith, H. 1981. Resistance of virulent and attenuated strains of Candida albicans to intracellular killing by human and mouse phagocytes. J. Infect. Dis.; 144: 557-565.

Salager, J.L. 2000. Formulation concepts for emulsion maker. In: Nielloud F, Mestres GM. editors pharmaceutical emulsion and suspension and pharmaceutical science; 19-68.

Schick, M.J. 1966. Nonionic surfactant, Marchel dekker In New york: 609-611.

Scudiero, D. A., Shoemaker, R. H., Paull, K. D., Monks, A., Tierney, S., Nofziger, T.H., Currens, M. J., Seniff, D., Boyd, M. R. 1988. Evaluation of soluble tetrazolium / Formazan Assay for cell growth and drug sensitivity in culture using human and other tumer cell lines. Cancer Res.; 48: 4827-4833.

Stone, H.D. 1988."Optimization of hydrophile-lipophile balance for improve efficacy of Newcastle disease and avian influenza oil-emulsion vaccines "Avian Dis.; 32(1): 68-73.

Stone, H.D., Brugh, M., Beard, C.W. 1980. Evaluation of inactivated Newcastle disease oil-emulsion vaccines. Avian Diseases 27: 688-697

Stone, H.D., Brugh, M., Bread, C.W. 1983. "influence of formulation on the efficacy of experimental oil-emulsion Newcastle disease vaccines" Avian Diseases., 27: 688-697.

Stone, H.D., Brugh, M., Hopkins, S.R., Yoder, H.W., Beard, C.W. 1978. Preparation of inactivated oil-emulsion vaccines with avian viral or mycoplasma antigens. Avian Dis., 22: 666-674.

Taha, M.M., Soad, M.S., Nadia, M.H., Salwa, E.A., Bakr, A.A. 1991. "Trails for preparation of formalin inactivated Newcastle disease oil emulsion vaccine in Egypt". Benha Vet.J., 1(2): 97-106.

Timms, L.M., Bracemell, C.D. 1983. " Cell mediated and humeral immune response of chicken $\mathrm{s}$ to inactivated oil emulsion infectious bronchitis vaccine ". Res. Vet. Si., 34: 224-230.

Wang, F., Zhao, Z., Li, X., Guan, P., Ri, M., Zhae, X., Gu, Y., Ji, L., Ma, X., Wu, Z., Cheng, Z. 1995. Studies Inactivated Oil-emulsion Vaccine against Avian Encephalomyelitis. Chinese journal of preventive veterinary medicine; $\mathrm{S}$ 852.5 .

Zheng, H., Guo, Y., Li, Z. 1995. Development of Inactivated Mixed Vaccine against ND and EDS-76 and Trial Observation on Growth and Decline Rate of Immune Antibody, Journal of animal infectious disease of china; S 852.4. 